\title{
Normal Midbrain Dopaminergic Neuron Development and Function in miR-133b Mutant Mice
}

\author{
Mary P. Heyer, ${ }^{1,2}$ Amar K. Pani, ${ }^{3}$ Richard J. Smeyne, ${ }^{3}$ Paul J. Kenny, ${ }^{2}$ and Guoping Feng ${ }^{1,4}$ \\ ${ }^{1}$ Department of Neurobiology, Duke University Medical Center, Durham, North Carolina 27710, ${ }^{2}$ Laboratory of Behavioral and Molecular Neuroscience, \\ Department of Molecular Therapeutics, The Scripps Research Institute Florida, Jupiter, Florida 33458, ${ }^{3}$ Department of Developmental Neurobiology, St. \\ Jude Children's Research Hospital, Memphis, Tennessee 38105, and ${ }^{4}$ McGovern Institute for Brain Research, Department of Brain and Cognitive Sciences, \\ Massachusetts Institute of Technology, Cambridge, Massachusetts 02139
}

Midbrain dopaminergic (mDA) neurons control movement and emotion, and their degeneration leads to motor and cognitive defects in Parkinson's disease (PD). miR-133b is a conserved microRNA that is thought to regulate mDA neuron differentiation by targeting Pitx3, a transcription factor required for appropriate development of $\mathrm{mDA}$ substantia nigra neurons. Moreover, miR-133b has been found to be depleted in the midbrain of PD patients. However, the function of miR-133b in the intact midbrain has not been determined. Here we show that miR-133b null mice have normal numbers of mDA neurons during development and aging. Dopamine levels are unchanged in the striatum, while expression of dopaminergic genes, including Pitx 3 , is also unaffected. Finally, motor coordination and both spontaneous and psychostimulant-induced locomotion are unaltered in miR-133b null mice, suggesting that miR-133b does not play a significant role in mDA neuron development and maintenance in vivo.

\section{Introduction}

Midbrain dopaminergic ( $\mathrm{mDA}$ ) neurons of the substantia nigra pars compacta $(\mathrm{SNpc})$ and ventral tegmental area (VTA) are critical for the regulation of motor function and emotion-related behaviors. mDA neurons are affected in several debilitating neurological disorders including Parkinson's disease (PD), which results from selective loss of SNpc DA neurons (Moore et al., 2005). While much progress has been made (Smidt and Burbach, 2007; Andressoo and Saarma, 2008; Cookson and Bandmann, 2010), the molecular mechanisms underlying the development and maintenance of mDA neurons and their loss in PD have not been fully elucidated.

Recently, microRNAs (miRNAs) have been implicated in mDA neuron differentiation and PD (Harraz et al., 2011). miRNAs are small, noncoding RNAs with diverse roles in nervous system development, function, and disease (Cao et al., 2006; Im and Kenny, 2012). miRNAs regulate gene expression by binding to the $3^{\prime}$-UTR of mRNA targets, decreasing mRNA

Received April 9, 2012; revised May 24, 2012; accepted June 1, 2012.

Author contributions: M.P.H., A.K.P., R.J.S., and G.F. designed research; M.P.H., A.K.P., and R.J.S. performed research; P.J.K. contributed unpublished reagents/analytic tools; M.P.H., A.K.P., R.J.S., and G.F. analyzed data; M.P.H. and G.F. wrote the paper.

This research was supported by a Ruth K. Broad Foundation Fellowship and a NINDS NRSA to M.P.H. G.F. was supported by NIH/NINDS and by The Poitras Center for Affective Disorders Research. We are grateful to Professor Marten Smidt of University Medical Center Utrecht for donation of the Pitx3 antibody. We thank Dr. Jacob Jacobsen of Duke University and Dr. Michael Cameron of The Scripps Research Institute Florida for advice related to in vivo microdialysis and HPLC-ECD.

The authors declare no competing financial interests.

Correspondence should be addressed to Dr. Guoping Feng, McGovern Institute for Brain Research, Department of

Brain and Cognitive Sciences, Massachusetts Institute of Technology, Building 46-3143, 43 Vassar Street, Cambridge, MA 02139. E-mail: fengg@mit.edu.

DOI:10.1523/JNEUROSCI.1732-12.2012

Copyright $\odot 2012$ the authors $\quad 0270-6474 / 12 / 3210887-08 \$ 15.00 / 0$ stability and protein translation (Bushati and Cohen, 2007). Deletion of the miRNA processing enzyme Dicer in postmitotic mDA neurons in mice causes extensive cell death, suggesting that miRNAs are critical for maintaining the $\mathrm{mDA}$ neuron population (Kim et al., 2007). One miRNA, miR-133b, was found to be enriched in the midbrain and specifically depleted in PD patients (Kim et al., 2007). In a mouse embryonic stem (ES) cell culture system, miR-133b negatively regulated ES cell differentiation into dopamine (DA) neurons. miR-133b has been proposed to mediate these effects by targeting Pitx3, a transcription factor required for differentiation of SNpc DA neurons; Pitx3 mutant mice lack SNpc DA neurons and display severe motor defects (van den Munckhof et al., 2003). miR-133b is a promising candidate in regulating $\mathrm{mDA}$ neuron differentiation and degeneration, but its role in vivo has not been determined.

Here we show that the mDA system develops normally in miR-133b null mice. mDA neuron morphology, number, and gene expression are unaltered early in development and in adult and aged miR-133b mutant mice. Whole-tissue levels of DA, norepinephrine (NE), and 5-hydroxytryptamine (5-HT) monoamine neurotransmitters in knock-out (KO) striatum are unchanged, while baseline and amphetamine-induced extracellular DA release are also unaffected. Furthermore, motor and anxiety-like behaviors in miR-133b null mice are equivalent to those of wild-type controls. From these data we conclude that miR-133b is not critical for mDA neuron development or function in vivo. In situ hybridization for mature miR-133b using locked nucleic acid (LNA) probes revealed modest expression in the $\mathrm{SNpc}$ as well as other brain regions, suggesting that miR-133b may have as yet undetermined functions in the mammalian nervous system. 

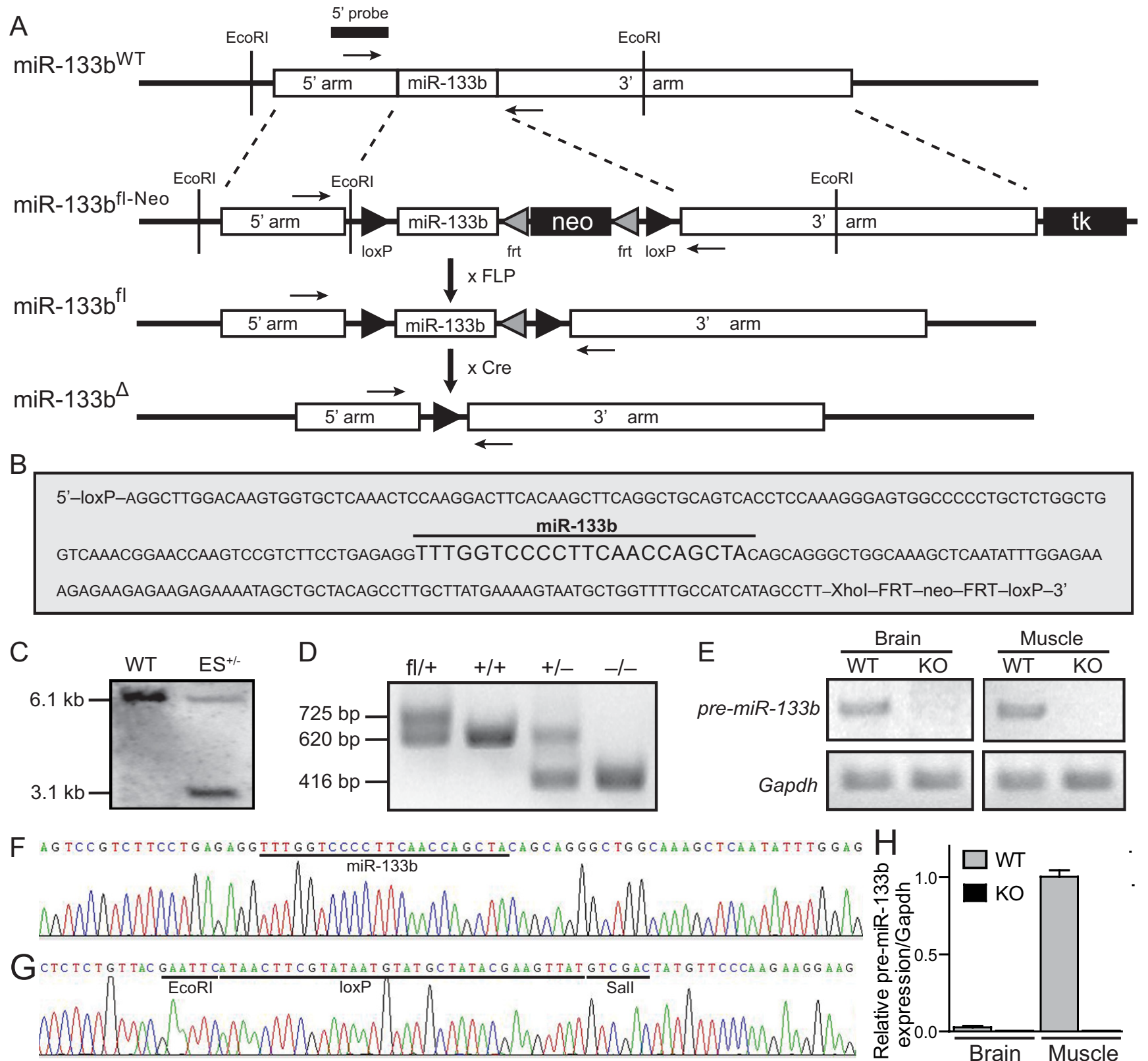
Cre deleter strain (The Jackson Laboratory) (Tallquist and Soriano, 2000) to create a miR-133-null allele, confirmed by PCR genotyping. miR-133b heterozygous mice were backcrossed to C57BL/ 6 for at least 8 generations, and all data were from wild-type (WT) and KO mice bred from miR-133b heterozygotes. Genotyping primers for detecting the floxed, WT, and null alleles were as follows: 133b-F, CAAGCTCTGTGAGAGGTTAGTCAGG; and 133b-R, CTCGACTGCATTTCCATTGTACTG (where $\mathrm{F}$ is forward and $\mathrm{R}$ is reverse). WT and KO bands were sequenced using the $133 \mathrm{~b}-\mathrm{F}$ and $133 \mathrm{~b}-\mathrm{R}$ primers to confirm deletion and correct orientation following loxP-mediated excision. Mice were housed 2-5 per cage in an Association for Assessment and Accreditation of Laboratory Animal Care-approved vivarium on a $12 \mathrm{~h}$ light-dark cycle. All experiments were carried out according to approved protocols from the Duke University and The Scripps Research Institute Florida Institutional Animal Care and Use Committees.

\section{Antibodies}

Mouse monoclonal anti-tyrosine hydroxylase $(\mathrm{TH})$ antibody $(1: 10,000, \mathrm{Im}-$ munostar) and rabbit anti-Pitx3 antibody (1:400, a gift from Dr. Marten Smidt, University Medical Center Utrecht, Utrecht, The Netherlands) were used for immunostaining and Western blot. Cy3-conjugated anti-rabbit antibody (1:400, Jackson ImmunoResearch) and Alexa Fluor 488-conjugated anti-mouse antibody (1:400, Invitrogen) were used for immunostaining. Mouse anti-GAD1 (1:1000, Millipore), goat anti-ChAT (1:1000, Millipore), anti- $\beta$-tubulin (1:5000, Sigma), anti- $\beta$-actin (1:5000, Sigma), anti-GAPDH (1:2000,Santa Cruz Biotechnology), and HRP-conjugated goat anti-mouse and anti-rabbit (1:5000, Jackson ImmunoResearch) antibodies were used for Western blot. Western blots of midbrain protein samples were performed using infra-red dye-conjugated secondary antibodies $(1: 10000, \mathrm{Li}-$ Cor) and a Li-Cor Odyssey infrared imager.

\section{Tyrosine hydroxylase immunohistochemistry}

Two-month-old male WT and miR-133b KO mice were deeply anesthetized with isoflurane (Butler Animal Health Supply) and transcardially perfused with physiological Ringer's solution followed by fixative (4\% paraformaldehyde in PBS). Whole brain was fixed overnight, equilibrated in $30 \%$ sucrose, and embedded in O.C.T. (Sakura). Thirty micrometer frozen coronal serial sections were collected using a cryostat (Leica CM1850). Heat-mediated antigen retrieval was performed by autoclaving in $1.8 \mathrm{~mm}$ citric acid and $8.2 \mathrm{~mm}$ sodium citrate. TH immunohistochemistry was performed as previously described (Johnson et al., 2010). Montage images were acquired using an AxioImager microscope fitted with a motorized stage controlled by the MosaiX functionality within AxioVision 5.1 software (Carl Zeiss MicroImaging).

\section{Unbiased stereology and cell counting}

Unbiased stereological estimation of the total number of TH immunoreactive (TH-IR) cell bodies in the SNpc and VTA was performed using the optical fractionator method, as described previously (Johnson et al., 2010). Every second section was collected through the midbrain and every fourth section was analyzed for cell counting, totaling 10 sections per animal. Cells at least $10 \mu \mathrm{m}$ in diameter stained for TH were classified as TH-IR. Embryonic day (E) 18.5 embryos were fixed in $4 \%$ PFA overnight at $4^{\circ} \mathrm{C}$, equilibrated in $30 \%$ sucrose, and embedded in O.C.T. Frozen $20 \mu \mathrm{m}$ coronal serial sections were collected throughout the midbrain region. After heatmediated antigen retrieval, sections were incubated with anti-TH and antiPitx 3 primary antibodies in 5\% normal goat serum, 2\% BSA, and $0.1 \%$ Triton X-100 in PBS overnight at $4^{\circ} \mathrm{C}$. Sections were incubated with Cy3 anti-rabbit and Alexa Fluor 488 anti-mouse antibodies and mounted with Fluoro-Gel (Electron Microscopy Sciences). Montage images were acquired of every second section throughout the rostral region of the SNpc, totaling five images per animal. All SNpc TH-IR, and Pitx3-IR cells were counted in each image using the counter tool in Photoshop (Adobe). All analyses were performed blind to genotype.

\section{Western blotting}

Three-month-old male and postnatal day $(\mathrm{P}) 0 \mathrm{WT}$ and $\mathrm{KO}$ mice were killed and whole brain or ventral midbrain dissected and flash frozen in liquid nitrogen. Tissue was homogenized in RIPA buffer (Sigma) supplemented with protease inhibitors (Complete, Roche). Twenty micro-
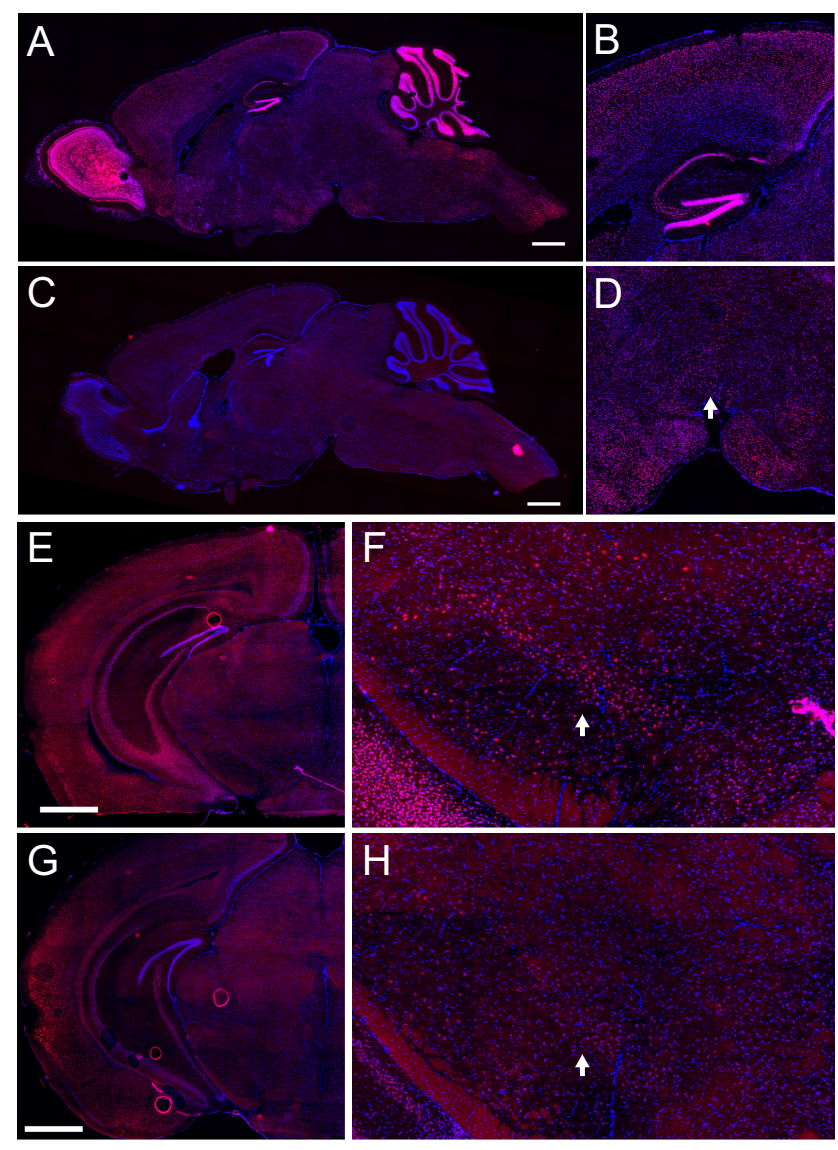

Figure 2. Detection of miR-133 expression in brain. $\boldsymbol{A}-\boldsymbol{H}$, Fluorescence in situ hybridization using antisense or scrambled LNA-DIG probes for mature miR-133b. Blue, DAPI staining of cell nuclei; Red, DIG detected by Cy3 signal following tyramide amplification. $\boldsymbol{A}$, miR-133b antisense probe signal in sagittal section of 3-month-old WT brain. $\boldsymbol{B}, \boldsymbol{D}$, Enlarged regions in $\boldsymbol{A}$ showing miR-133b signal in cortex and hippocampus $(\boldsymbol{B})$ and ventral midbrain (arrow) and neighboring regions (hypothalamus, pons) (D). C, Signal from scrambled miR-133b LNA-DIG probe. $\boldsymbol{E}, \boldsymbol{F}$, Coronal section through midbrain region showing miR-133 signal in substantia nigra pars compacta region, indicated by arrow in inset $(\boldsymbol{F}) . \mathbf{G}, \boldsymbol{H}$, Coronal section through 3-month-old miR133b KO midbrain region with detectable miR-133 signal in SNpc, indicated by arrow in inset $(\boldsymbol{H})$, likely due to miR-133a1 and miR-133a2 expression. Scale bars, $1 \mathrm{~mm}$.

grams of lysate were run on $10-12 \%$ SDS-polyacrylamide gels, and Western blotting was carried out according to standard procedures. Bands of whole brain Western blots were visualized using ECL Plus (GE Healthcare Life Sciences) and a LAS-3000 Intelligent Dark Box (Fujifilm). Bands of midbrain Western blots were visualized using a Li-Cor Odyssey infrared imager. Bands were quantified by densitometry analysis using ImageJ software and normalized to $\beta$-actin (for TH and GAD1), $\beta$-tubulin (for Pitx3) or GAPDH (midbrain samples).

\section{RNA extraction and real-time PCR}

Three-month-old male and PO WT and KO mice were sacrificed and whole brain was flash frozen in liquid nitrogen. Total RNA was extracted using TRIzol reagent (Invitrogen) and treated with Turbo DNase (Ambion). One microgram of RNA was used for reverse transcription with oligo-dT and random primers and AffinityScript enzyme (Stratagene). cDNA was used for quantitative PCR (Power SYBR Green, ABI 7300 real-time PCR machine, Applied Biosystems). The following intronspanning primers were used: Gapdh-F, CATGGCCTTCCGTGTTCCT; Gapdh-R, TGATGTCATCATACTTGGCAGGTT; TH-F, CTCTCCACGGTGTACTGGTTCAC; TH-R, GGCTGGTAGGTTTGATCTTGGTAG; DAT-F, GTATGTGGTCGTGGTCAGCATT; DAT-R, CTTTCTCAGGTGTGATGGCATAG; GAD1-F, CAACCAGATGTGTGCAGGCTAC; GAD1-R, GCGTAGAGGTAATCAGCCAGCTC; ChAT-F, CTTTTGTGCAAGCCATGACTGAC; ChAT-R, CATCCATGAACATCTCAGGTGG; 
Pitx3-F, CAGAGGAATCGCTACCCTGACA; Pitx3-R, AAGCCACCTTTGCACAGCTC. Primers amplifying the miR-133al stem loop were as follows: miR-133a1-F, GGTTGACAGTTGCTAGGTATTTGC; and miR-133a1-R, GCTTTGCTAAAGCTGGTAAAATGG. Whole brain cDNA was also used for standard PCR amplification and quantitative PCR to confirm loss of the miR-133b stem loop in KO samples using the following primers: $m i R-133 b$-F, AGGCTTGGACAAGTGGTGCTCAA; and miR-133b-R, AAGGCTATGATGGCAAAACCAGC. Each sample was measured in triplicate and normalized to expression of Gapdh.

\section{Neurochemical analysis of whole tissue}

Two-month-old male WT and miR-133b KO mice were deeply anesthetized with isoflurane and sacrificed by decapitation. Frontal cortex, nucleus accumbens, dorsal striatum, and thalamus were rapidly dissected on a cold plate, flash-frozen in liquid nitrogen, and stored at $-80^{\circ} \mathrm{C}$ for high performance liquid chromatography with electrochemical detection (HPLCECD) analysis. A modified chromatographic procedure was employed, as described previously (Pani, 2009). All procedures were performed blind to genotype.

\section{In vivo microdialysis}

Ten-week-old male WT and miR-133b KO mice were deeply anesthetized with tribromoethanol and a stereotaxic apparatus (David Kopf Instruments) used to intracranially implant microdialysis guide cannulae (CMA7, CMA Microdialysis) in dorsal striatum (coordinates in mm: 0.5 anteroposterior, $2.0 \mathrm{me}-$ diolateral, -1.5 dorsoventral from bregma). Following recovery for 3-5 days, a microdialysis probe (CMA7, $2 \mathrm{~mm}$ membrane length, CMA Microdialysis) was inserted into the guide cannula and perfused overnight with artificial CSF (aCSF perfusion fluid: $145 \mathrm{~mm} \mathrm{NaCl}, 3.5 \mathrm{~mm} \mathrm{KCl}, 2 \mathrm{~mm}$ $\mathrm{Na}_{2} \mathrm{HPO}_{4}, 1 \mathrm{~mm} \mathrm{CaCl}, 1.2 \mathrm{~mm} \mathrm{MgCl}$, pH 7.4, CMA Microdialysis) at a flow rate of $0.45 \mu \mathrm{l} / \mathrm{min}$ (CMA Microdialysis 400 syringe pump). The following day, the flow rate was increased to $1 \mu \mathrm{l} / \mathrm{min}$ for 1 hour before sample collection. Dialysate samples were collected at 20 min intervals into vials containing $10 \% 0.1 \mathrm{~m}$ perchloric acid to prevent oxidation of monoamines. Three baseline samples were collected, $100 \mu \mathrm{M}$ amphetamine in aCSF was delivered to the probe by reverse microdialysis, and four more samples were collected to assess amphetamine-induced neurotransmitter release. Animals were kept on a reverse light-dark cycle, and all microdialysis samples were collected during the dark-active phase from freely moving animals. Mice were deeply anesthetized with isoflurane and transcardially perfused, brains were removed and embedded in O.C.T., and frozen coronal sections were collected from striatum to confirm probe placement.

\section{Liquid chromatography and electrochemical detection}

Dopamine contents from dialysates were quantified by HPLC-ECD. Ten microliters of each sample was automatically injected using an Agilent 1100 liquid chromatography apparatus and run on an MD-150 HPLC column $(3 \times 150 \mathrm{~mm}, 3 \mu \mathrm{m}$ particle size). MD-TM mobile phase (ESA) was delivered at a flow rate of $0.6 \mathrm{ml} / \mathrm{min}$. Following column separation, monoamines were detected using an ESA 5021A guard cell, an ESA $5011 \mathrm{~A}$ analytical cell, and an ESA Coulochem III detector $\left(E_{\mathrm{GC}}=+350\right.$ $\mathrm{mV}, E_{1}=-150 \mathrm{mV}, E_{2}=+220 \mathrm{mV}$; filter time constant, $5 \mathrm{~s}$ ). Characteristic retention times of dihydroxyphenylacetic acid, dopamine, and homovanillic acid were 3.5, 4.3, and 7.2 min, respectively. Analyte peak areas were integrated and interpolated from known standards using Agi- lent ChemStation software and GraphPad software. All procedures were carried out blind to genotype.

\section{Behavioral analyses}

Open field. The open field test was performed as described previously (Peça et al., 2011). Briefly, 3- to 4-month-old WT and KO mice were placed in automated $16 \times 16$ inch chambers (AccuScan Instruments) for $60 \mathrm{~min}$. Total distance traveled (centimeters) and time spent in the center were detected in $5 \mathrm{~min}$ bins. Two weeks later, the same mice were placed in $8 \times 8$ inch chambers, and distance traveled was similarly detected for $60 \mathrm{~min}$. Three milligrams per kilogram of amphetamine was immediately administered by i.p. injection, the mice were returned to their chambers, and locomotor activity was recorded for a further $120 \mathrm{~min}$.

Zero maze. Five-month-old WT and KO mice were placed in the closed arm of an elevated zero maze indirectly illuminated at 100 lux, and their behavior was videotaped over $5 \mathrm{~min}$ (Peça et al., 2011). The time spent in the closed and open arms was scored blind to genotype using The Observer software (Noldus).

Dark-light emergence. The dark-light emergence task was carried out as described previously (Peça et al., 2011). Three- to four-month-old WT and $\mathrm{KO}$ mice were tested in an automated dual chamber apparatus (Med Associates) containing a gate between a "light" (1000 lux) and "dark" chamber. The mice were placed in the dark chamber and the gate lifted, allowing the mice to freely move between both chambers. The duration in the light and dark chambers was recorded over $5 \mathrm{~min}$.

Marble burying. Marble burying behavior of 5-month-old male WT and $\mathrm{KO}$ mice was assessed (Deacon, 2006). Twenty-four $2 \mathrm{~cm}$ diameter marbles were evenly spaced on the surface in an 8.5 inch $\times 16$ inch chamber filled 5 inches deep with aspen shavings. A mouse was placed in 
one corner of the chamber and allowed to move freely. After $30 \mathrm{~min}$, the mouse was removed and an image taken with a digital camera (Canon). The number of visible marbles was counted, and the remainder recorded as the number of marbles buried.

Rotarod. Five-month-old male WT and KO mice were tested on the rotarod task (Jones and Roberts, 1968). Mice were trained for four trials per day over 3 days on a rotarod device (Med Associates) at a steady speed of $24 \mathrm{rpm}$. The latency to fall was recorded as the time at which the mouse fell completely off the rod or failed to stay atop the rod. The intertrial interval was $5 \mathrm{~min}$. On the fourth day the mice underwent an accelerating rotarod task, where rotation velocity increased from 4 to $40 \mathrm{rpm}$ over 5 min. Performance on the task was assessed by measuring the average latency to fall off the rod over three trials.

\section{miRNA fluorescence in situ hybridization}

Fluorescence in situ hybridization for miR-133b was performed on 16 $\mu \mathrm{m}$ sagittal sections of 3-month-old WT and KO mouse brains according to a previously described method using 1-ethyl-3-(3-dimethylaminopropyl)carbodiimide fixative to prevent loss of miRNAs (Pena et al., 2009). LNA oligonucleotides (Exiqon) were labeled with digoxigenin using a 3 '-end labeling kit (Roche). In situ hybridization was carried out with a miR-133b antisense probe, a miR-124 antisense probe as a positive control, and a scrambled miR-133b probe as a negative control. Images were acquired with an Olympus BX51 microscope and the Virtual Tissue module of Stereo Investigator software.

\section{Statistical analyses}

All data were analyzed using Prism software (GraphPad Software). Stereological cell counts, mRNA and protein expression data, zero maze data, dark-light emergence test data, and rotarod data were analyzed by an unpaired two-tailed $t$ test. Tissue monoamine levels were analyzed by one-way ANOVA, and microdialysis and open field data were analyzed by repeated-measures ANOVA followed by Bonferroni post hoc test. Data are presented as mean plus SEM.

\section{Results}

\section{Generation of miR-133b mutant mice}

miR-133b is embedded in an intergenic noncoding gene, 7H4, located on chromosome 1 (Velleca et al., 1994). loxP sites were placed around the miR-133b stem loop precursor region, and a FRT-flanked pGK-Neo cassette was inserted 3 ' to the floxed stem loop (Fig. $1 A, B$ ). The targeting vector was introduced into ES cells, and homologous recombination at the miR-133b locus was detected by Southern blot (Fig. 1C). Correctly targeted ES cells were used to generate chimeric mice, and germline transmission of the targeted allele was confirmed by PCR screening. The Neo cassette was excised by mating miR-133 $\mathrm{b}^{\text {flox-Neo/+ }}$ mice with a FLPe deleter strain, and subsequent miR-133 $\mathrm{b}^{\text {flox/+ }}$ mice were mated with a Cre deleter strain to remove the miR-133b stem loop. Floxed, WT, heterozygous, and $\mathrm{KO}$ mouse genotypes were detected by PCR of tail DNA (Fig. 1D), and WT and KO PCR products were sequenced to detect deletion of $\mathrm{miR}-133 \mathrm{~b}$ and presence of a single loxP site in the $\mathrm{KO}$ (Fig. $1 F, G$ ). Loss of miR-133b was confirmed by RT-PCR and quantitative RT-PCR for the miR-133b stem loop region (Fig. $1 E, H$ ). Heterozygous mice were backcrossed at least eight generations to C57BL/6 mice and maintained on a C57BL/6 background. miR-133b KO mice were viable, fertile, and born at a Mendelian ratio. The $\mathrm{KO}$ mice appeared grossly normal, had similar weights and lifespan as their WT littermates, and did not display any overt behavioral defects.

\section{Detection of miR-133b in brain by fluorescence in situ hybridization}

miR-133b has previously been found to be enriched in midbrain; however, its precise expression pattern has not been determined. We performed fluorescence in situ hybridization (FISH) for ma-
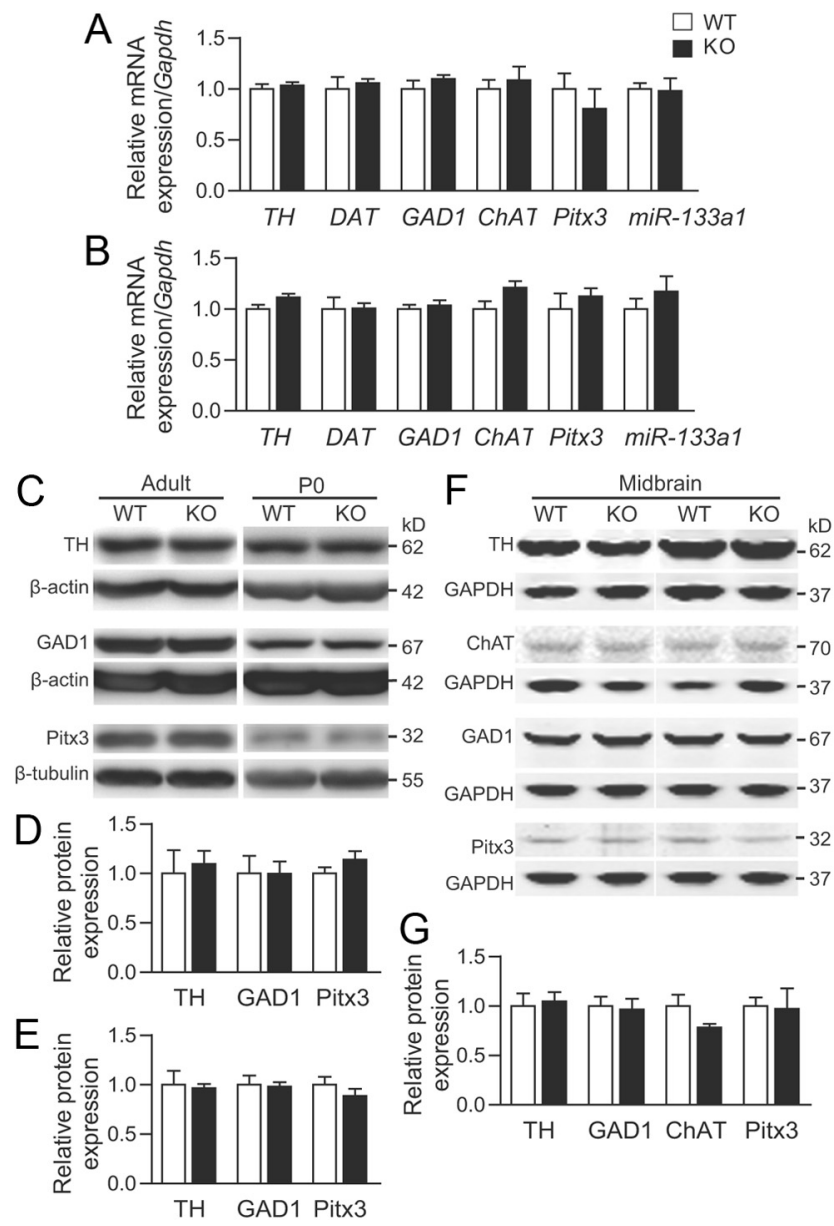

Figure 4. Unaltered dopaminergic gene expression in miR-133b K0 mice. $\boldsymbol{A}, \boldsymbol{B}, T H, D A T$ GAD1, ChAT, Pitx3, and miR-133a1 mRNA expression in whole brain of 3-month-old male $(\boldsymbol{A})$ and PO (B) WT and miR-133b KO mice. Gene expression is normalized to Gapdh and results are scaled to the average value of the WT samples (WT average, $1 ; n=4$ WT and $4 \mathrm{KO}$ mice). C, TH, GAD1, and Pitx3 protein expression in whole brain of 3-month-old male (quantified in D) and PO (quantified in $\boldsymbol{E}$ ) WT and miR-133b KO mice. Western blot bands were quantified by densitometry and normalized to $\beta$-actin (for TH and GAD1) or $\beta$-tubulin (for Pitx3). Results were scaled to the average value of the WT samples (WT average, $1 ; n=4$ WT and 4 KO mice). $F$, TH, ChAT GAD1, and Pitx3 protein expression in ventral midbrain of WT and miR-133b KO mice. G, Western blot bands in $\boldsymbol{F}$ were quantified in by densitometry and normalized to GAPDH. Results were scaled to the average value of the WT samples (WT average, $1 ; n=5$ WT and 6 K0 mice). Error bars indicate SEM.

ture miR-133b in adult brain using LNA modified, digoxigenin (DIG)-labeled antisense and scrambled probes (Fig. 2). miR133b signal could be observed in many different brain regions, particularly cortex, hippocampus, and cerebellum (Fig. $2 A, B$ ), as well as regions neighboring the midbrain such as hypothalamic and pontine nuclei (Fig. $2 A, D$ ). Lack of signal from a miR-133b scrambled probe suggested that these regions were not stained due to nonspecific binding (Fig. 2C). Consistent with previous reports of biochemical enrichment in midbrain, we did detect moderate miR-133b expression in the SNpc region of ventral midbrain (Fig. 2 E, F). However, some signal from the miR-133b antisense probe could still be detected in miR-133b KO brain, including the SNpc (Fig. 2G,H). The orthologs of miR-133b, miR-133a1, and miR-133a2 only differ by one base from miR$133 \mathrm{~b}$ and, although not found to be enriched, have also been biochemically detected in brain and midbrain. Thus, signal from the miR-133b antisense probe in various regions of miR-133b KO 
brain is likely due to binding to the remaining miR-133 family members.

Detection of miR-133b expression in SNpc by FISH, together with previous findings that miR-133b is enriched in midbrain and expressed in ES cell-derived DA neurons in vitro, warranted further investigation into its function in the midbrain dopaminergic system in vivo.

The midbrain is morphologically unchanged in miR-133b mutant mice miR-133b has been shown to negatively regulate dopaminergic neuron differentiation in cultured mouse ES cells (Kim et al., 2007); therefore, miR-133b KO mice might be expected to have altered numbers of mDA neurons. The general morphology of the midbrain and striatum of 2-month-old male KO mice was indistinguishable from those of WT mice (Fig. $3 A, B)$, while morphology and unbiased stereological counts of TH-IR cells in VTA and $\mathrm{SNpc}$ were also unchanged (Fig. $3 A, D)$. miR-133b is selectively depleted in the midbrain of PD patients; if loss of miR-133b contributes to PD pathogenesis, deletion of miR-133b could lead to decreased numbers of DA neurons in aged mice. We found no difference in midbrain TH-IR cell number in 12-month-old WT and KO animals (Fig. $3 C, F)$, suggesting that loss of miR-133b does not cause degeneration of $\mathrm{mDA}$ neurons. To determine whether $\mathrm{KO}$ mice have abnormal DA neuron development that is corrected postnatally, TH-IR cells were counted in E18.5 WT and KO SNpc. No difference was observed in number or morphology of TH-IR cells (Fig. $3 C, F)$, consistent with normal development of DA neurons. A small increase in the number of Pitx3-IR cells was observed in the KO (Fig. 3F), suggesting that miR-133b may target Pitx3 in vivo. However, the number of cells immunoreactive for both $\mathrm{TH}$ and Pitx 3 was equivalent in $\mathrm{WT}$ and $\mathrm{KO}$ (Fig. $3 F$ ), suggesting that the increased number of Pitx3-expressing cells in KOs does not have a major influence on $\mathrm{mDA}$ cell fate.

\section{Midbrain dopaminergic neuron gene expression is unaffected} in miR-133b mutant mice

While miR-133b does not appear to regulate the differentiation of DA neurons in vivo, it could act by regulating expression of genes that affect neuron function but not cell number. Therefore, we quantified the expression levels of several genes important for mDA neuron function. TH, DAT, and Pitx 3 transcripts were at WT levels in neonatal and adult KO brain, and levels of GAD1 and $C h A T$, expressed in GABAergic and cholinergic midbrain neurons, respectively, were also unchanged (Fig. $4 A, B$ ). Furthermore, expression of the related miRNA, miR-133al, was unaltered in KO brain. Since many miRNAs act post-transcriptionally, miR133b deletion could affect protein expression without altering transcript levels. However, no difference in neonatal or adult brain TH, GAD1, or Pitx3 protein levels was observed between WT and $\mathrm{KO}$ animals (Fig. 4C-E). To more specifically determine whether dopaminergic, GABAergic or cholinergic neurons had altered protein expression in midbrain, TH, GAD1, ChAT, and Pitx3 levels were quantified in WT and KO ventral midbrain, and
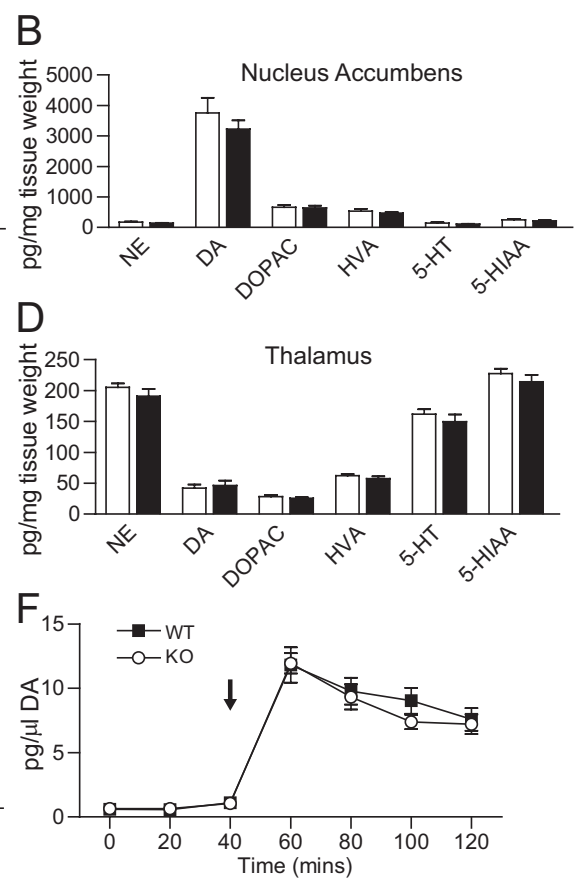

Frontal Cortex

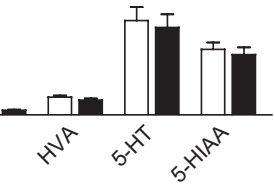

Hypothalamus

5. Unchanged monoamine neurotransmitter levels in miR-133b KO mice. $\boldsymbol{A}-\boldsymbol{E}$, Neurochemical analysis of whole-tissue -month-old male WT and miR-133b K0 mice; $n=8$ WT and 7 K0 mice. $F$, Neurochemical analysis of baseline and amphetaminestimulated extracellular DA levels in dorsal striatum of 3-month-old male WT and KO mice. Amphetamine at $100 \mu \mathrm{m}$ was continually infused via reverse microdialysis after $40 \mathrm{~min}$ (arrow); $n=9 \mathrm{WT}$ and $8 \mathrm{KO}$ mice. Error bars indicate SEM.

no difference was observed (Fig. $4 F, G$ ), suggesting that global expression patterns in brain and midbrain are not affected by loss of miR-133b.

\section{Monoamine neurotransmitters are unaltered in miR-133b mutant mice}

To evaluate whether the activity of mDA neurons was altered in miR-133b KO mice, whole tissue monoamine neurotransmitter levels were quantified by HPLC-ECD. Levels of DA, NE, 5-HT, and their metabolites were unchanged in dorsal striatum (Fig. $5 A$ ), nucleus accumbens (Fig. $5 B$ ), frontal cortex (Fig. 5C), thalamus (Fig. 5D), and hypothalamus (Fig. $5 E$ ) of KO mice. To determine whether extracellular DA release was altered following miR-133b deletion, baseline and amphetamine-stimulated dorsal striatal microdialysis perfusates were collected from 3-monthold WT and KO male mice, and DA levels were quantified by HPLC-ECD. Baseline and amphetamine-induced DA release was equivalent between WT and KO samples, with both exhibiting a similar increase in extracellular DA upon administration of amphetamine ( $\sim 20$-fold above baseline), followed by a gradual decline (Fig. 5F). These data suggest that miR-133b does not regulate total DA neurotransmitter production in $\mathrm{mDA}$ neurons or DA release in dorsal striatum.

Locomotor and anxiety-like behaviors in miR-133b mutants are similar to those of wild-type mice

Movement and motivation-related behaviors are directly linked to $\mathrm{mDA}$ neuron function. To determine whether miR-133b KO mice have an underlying defect in the midbrain dopaminergic system, mice underwent a battery of behavioral tests. $\mathrm{KO}$ mice exhibited spontaneous locomotor behavior in the open field test that was indistinguishable from that of WT mice (Fig. 6A). The response of $\mathrm{KO}$ mice after injection of the psychostimulant am- 

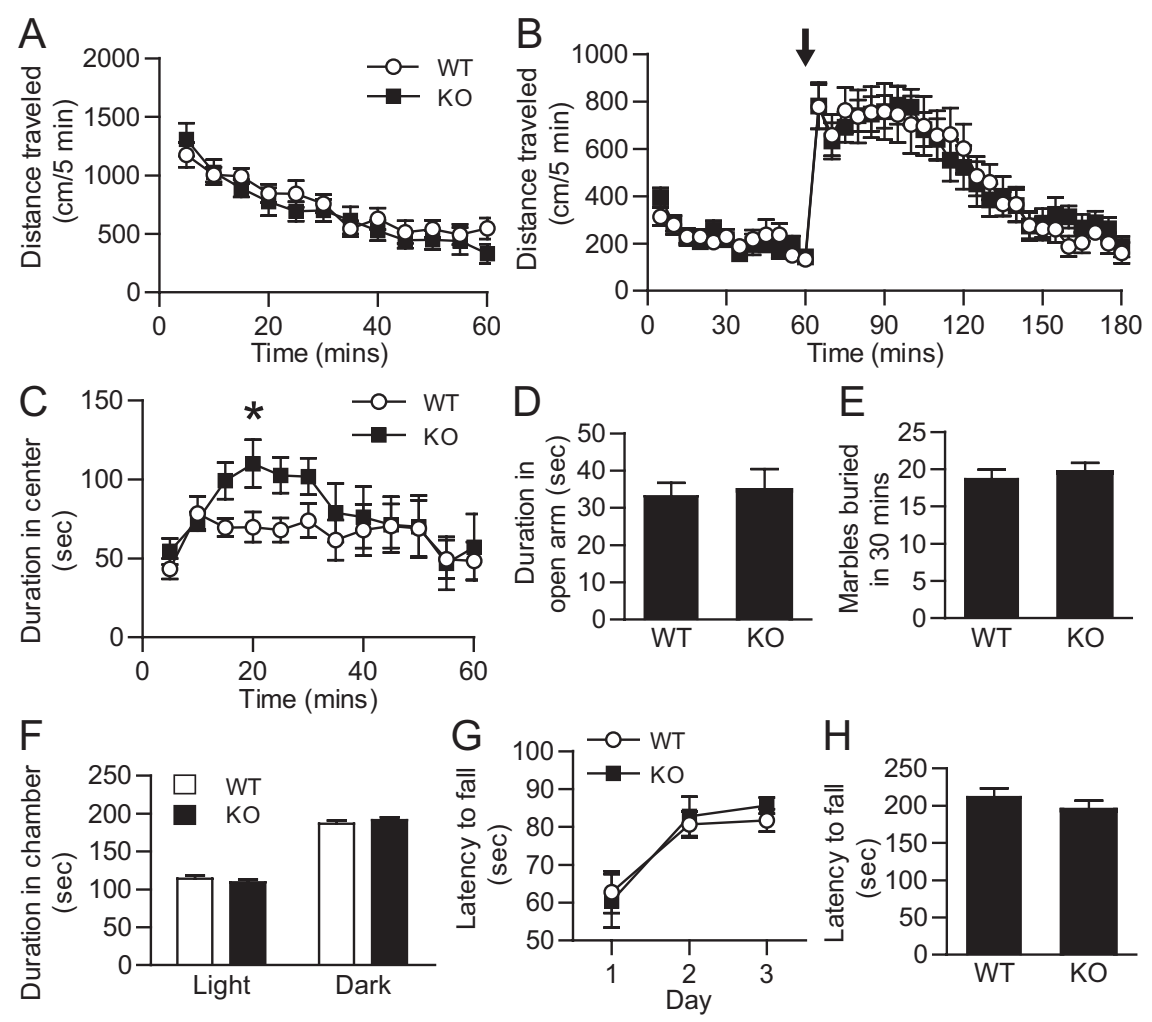

Figure 6. Motor and anxiety-like behaviors are similar in miR-133b WT and K0 mice. $\boldsymbol{A}$, Spontaneous locomotor activity of 3-to 4-month-old WT and KO mice in the open field test; $n=12$ WT and $12 \mathrm{KO}$ mice. $\boldsymbol{B}$, Amphetamine-induced locomotor activity of 3to 4-month-old WT and KO mice in the open field test. Three milligrams per kilogram of amphetamine was administered by i.p. injection at $60 \mathrm{~min}$ (arrow); $n=9$ WT and $11 \mathrm{KO}$ mice. $C$, miR-133b KO mice spend more time in the center of the open field chamber during the first 30 min of the open field test; ${ }^{*} p<0.05$, Student's $t$ test. $n=12$ WT and 12 K0 mice. $D$, Time spent by 3to 4-month-old WT and K0 mice in the open arm of the elevated plus zero maze over 5 min; $n=18$ WT and 13 KO mice. $\boldsymbol{E}$, Number of marbles buried beneath bedding by 5 -month-old male WT and K0 mice after 30 min; $n=13$ WT and 9 KO mice. $F$, Time spent in the light versus dark chamber by 3- to 4-month-old WT and K0 mice in the dark-light emergence test over $5 \mathrm{~min} ; n=15 \mathrm{WT}$ and $10 \mathrm{KO}$ mice. G, Average latency to fall of 5 -month-old male WT and $\mathrm{KO}$ trained on a rotarod at a fixed speed of $24 \mathrm{rpm}$ for 4 trials per day over 3 days. $\boldsymbol{H}$, Latency to fall from an accelerating rotarod $(4-40 \mathrm{rpm})$ on the fourth day, averaged over 3 trials; $n=13$ WT and $9 \mathrm{KO}$ mice. Error bars indicate SEM.

phetamine, which elevates extracellular DA levels, was also similar to that of WT mice (Fig. 6 B). KO mice spent more time in the center of the open field during the first $30 \mathrm{~min}$ of the test as compared to WT mice (Fig. 6C), suggesting a decrease in anxietylike behavior. mDA neuron dysfunction in mice has been found to cause generalized anxiety (Zweifel et al., 2011); to test whether $\mathrm{KO}$ mice were less anxious, behavior was observed in the elevated zero maze test (Fig. 6D). KO mice exhibited anxiety-like behavior equivalent to that of WT, preferring to spend less time in the open arms of the maze. The behavior of $\mathrm{KO}$ mice did not differ from that of WT in the marble burying test (Fig. 6E) or dark-light emergence test (Fig. $6 F$ ), which are both tests of anxiety-like behavior. Finally, motor coordination and learning were evaluated in the rotarod test. Mice were trained over three days to stay on top of a rod rotating at a fixed velocity, and $\mathrm{KO}$ mice learned the task as easily as their WT counterparts (Fig. 6G). Motor coordination was tested further on the fourth day as the rod accelerated to its maximum velocity. The performance of $\mathrm{KO}$ mice was equivalent to that of WT mice, as assessed by the duration of their attempt to stay on the rod (Fig. $6 H$ ). Therefore, miR-133b KO mice appear to have normal motor and anxiety-like behaviors, consistent with appropriate midbrain dopaminergic neuron function.

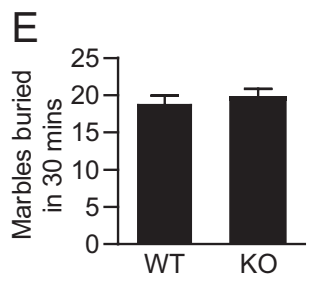

\section{Discussion}

Here we show that mice with a deletion of miR-133b, a miRNA previously proposed to regulate differentiation and survival of mDA neurons (Kim et al., 2007), display no differences in development or maintenance of mDA neurons. We could detect no significant abnormalities in $\mathrm{mDA}$ neuron morphology, cell number, gene expression, monoamine levels, or motor and anxiety-like behaviors in these mice. These data suggest that the function of miR-133b in an in vitro model of DA neuron differentiation does not translate to a similar role in the intact animal. There are several explanations for this discrepancy. First, miR-133b expression in the midbrain may not contribute significantly to $\mathrm{mDA}$ neuron function. Indeed, $\mathrm{miR}-133 \mathrm{~b}$ is part of the miR-133 family of miRNAs that is highly expressed in muscle and has been more extensively characterized in skeletal muscle development (TownleyTilson et al., 2010). Overexpression of muscle miRNAs in heterologous cells globally inhibits brain-specific gene expression (Lim et al., 2005); introduction of miR-133b in a neuron culture system could be expected to inhibit neuronal differentiation in a broad manner without having a specific role in DA neurons.

Second, while not found to be enriched, expression of the miR-133b paralogs miR-133a1 and miR-133a2 has also been detected in the midbrain (Kim et al., 2007) and could compensate for the loss of miR-133b. miR-133al expression was not altered in miR-133b KO brain, while miR-133a2 expression was below our detection limits. Therefore, the lack of miR133b does not appear to feed back to increase expression of its paralogs, although we cannot rule out the possibility of compensation by existing levels of miR-133a in midbrain. miR-133al and miR-133a2 double KO mice have recently been reported (Liu et al., 2008). It will now be possible to generate mice with a triple deletion of miR-133a1, miR-133a2, and miR-133b to determine whether the miR-133 family of miRNAs is important for brain development and function.

We have found that miR-133b is expressed in the SNpc region of midbrain, in addition to many other brain regions, including cortex, hippocampus, and cerebellum. Recently, cell type-specific miRNA biochemical purification combined with deep sequencing has shown that miR-133b is expressed in GABAergic neurons of cortex and cerebellum (He et al., 2012). This method will be critical for teasing apart the specific expression patterns of miR133b, miR-133al, and miR-133a2 in various neuronal subtypes and brain regions. The specific functions of miR-133b in these cell types may subsequently be explored using the constitutive or conditional miR-133b knock-out mouse lines described here.

There is growing evidence that miRNAs are implicated in neurodegenerative disorders (Hébert and De Strooper, 2009), presenting new possibilities for therapeutic intervention (Seto, 
2010). miR-133b depletion in the midbrain of PD patients led to the hypothesis that loss or dysfunction of miR-133b could promote PD progression (Kim et al., 2007). However, we did not detect $\mathrm{mDA}$ neuron degeneration or impaired motor function in aged miR-133b KO animals. Moreover, no association has been found between miR-133b genetic variants and PD (de Mena et al., 2010). These results suggest that miR-133b depletion may not be causal in PD neurodegeneration; rather, loss of $\mathrm{mDA}$ neurons during disease progression would necessarily lead to a reduction in levels of miR-133b, along with other genes expressed in $\mathrm{mDA}$ neurons. However, we cannot exclude the possibility that a decrease in miR-133b may enhance the susceptibility of mDA neurons to neurodegeneration resulting from $\mathrm{PD}$ or toxic insults. This could be tested in the future by comparing PD-like disease progression in WT and miR-133b KO mice following MPTP or 6-OHDA treatment. Although miR-133b does not appear to play a causal role in midbrain neurodegeneration, loss of all miRNAs in mDA neurons in vivo does cause profound neuronal cell death (Kim et al., 2007). Therefore, other miRNAs expressed in mDA neurons may act alone or in concert to promote neuronal development and survival. In vivo analyses of these miRNAs may pave the way for novel therapeutic strategies in PD and other disorders of the mDA system.

\section{References}

Andressoo JO, Saarma M (2008) Signalling mechanisms underlying development and maintenance of dopamine neurons. Curr Opin Neurobiol 18:297-306.

Bushati N, Cohen SM (2007) microRNA functions. Annu Rev Cell Dev Biol 23:175-205.

Cao X, Yeo G, Muotri AR, Kuwabara T, Gage FH (2006) Noncoding RNAs in the mammalian central nervous system. Annu Rev Neurosci 29: 77-103.

Cookson MR, Bandmann O (2010) Parkinson's disease: insights from pathways. Hum Mol Genet 19:R21-R27.

Deacon RM (2006) Digging and marble burying in mice: simple methods for in vivo identification of biological impacts. Nat Protoc 1:122-124.

de Mena L, Coto E, Cardo LF, Díaz M, Blázquez M, Ribacoba R, Salvador C, Pastor P, Samaranch L, Moris G, Menéndez M, Corao AI, Alvarez V (2010) Analysis of the Micro-RNA-133 and PITX3 genes in Parkinson's disease. Am J Med Genet B Neuropsychiatr Genet 153B:1234-1239.

Farley FW, Soriano P, Steffen LS, Dymecki SM (2000) Widespread recombinase expression using FLPeR (flipper) mice. Genesis 28:106-110.

Harraz MM, Dawson TM, Dawson VL (2011) MicroRNAs in Parkinson's disease. J Chem Neuroanat 42:127-130.

He M, Liu Y, Wang X, Zhang MQ, Hannon GJ, Huang ZJ (2012) Cell-typebased analysis of microRNA profiles in the mouse brain. Neuron 73:35-48.

Hébert SS, De Strooper B (2009) Alterations of the microRNA network cause neurodegenerative disease. Trends Neurosci 32:199-206.

Heyer MP, Feliciano C, Peça J, Feng G (2011) Elucidating gene function through use of genetically engineered mice. In: Genomics: essential meth- ods (Starkey MP, Elaswarapu R, eds), pp 211-248. Oxford: Wiley-Blackwell.

Im HI, Kenny PJ (2012) MicroRNAs in neuronal function and dysfunction. Trends Neurosci 35:325-334

Johnson ML, Ho CC, Day AE, Walker QD, Francis R, Kuhn CM (2010) Oestrogen receptors enhance dopamine neurone survival in rat midbrain. J Neuroendocrinol 22:226-237.

Jones BJ, Roberts DJ (1968) The quantiative measurement of motor in coordination in naive mice using an acelerating rotarod. J Pharm Pharmacol 20:302-304

Kim J, Inoue K, Ishii J, Vanti WB, Voronov SV, Murchison E, Hannon G, Abeliovich A (2007) A MicroRNA feedback circuit in midbrain dopamine neurons. Science 317:1220-1224.

Lim LP, Lau NC, Garrett-Engele P, Grimson A, Schelter JM, Castle J, Bartel DP, Linsley PS, Johnson JM (2005) Microarray analysis shows that some microRNAs downregulate large numbers of target mRNAs. Nature 433:769-773.

Liu N, Bezprozvannaya S, Williams AH, Qi X, Richardson JA, Bassel-Duby R, Olson EN (2008) microRNA-133a regulates cardiomyocyte proliferation and suppresses smooth muscle gene expression in the heart. Genes Dev 22:3242-3254.

Moore DJ, West AB, Dawson VL, Dawson TM (2005) Molecular pathophysiology of Parkinson's disease. Annu Rev Neurosci 28:57-87.

Nagy A, Rossant J, Nagy R, Abramow-Newerly W, Roder JC (1993) Derivation of completely cell culture-derived mice from early-passage embryonic stem cells. Proc Natl Acad Sci U S A 90:8424-8428.

Pani AK (2009) Influence of intrastriatal infusion of dynorphin fragments on overflow of acetylcholine and dopamine in the rat brain. Int J Neurosci 119:1362-1383.

Peça J, Feliciano C, Ting JT, Wang W, Wells MF, Venkatraman TN, Lascola CD, Fu Z, Feng G (2011) Shank3 mutant mice display autistic-like behaviours and striatal dysfunction. Nature 472:437-442.

Pena JT, Sohn-Lee C, Rouhanifard SH, Ludwig J, Hafner M, Mihailovic A, Lim C, Holoch D, Berninger P, Zavolan M, Tuschl T (2009) miRNA in situ hybridization in formaldehyde and EDC-fixed tissues. Nat Methods 6:139-141

Seto AG (2010) The road toward microRNA therapeutics. Int J Biochem Cell Biol 42:1298-1305.

Smidt MP, Burbach JP (2007) How to make a mesodiencephalic dopaminergic neuron. Nat Rev Neurosci 8:21-32.

Tallquist MD, Soriano P (2000) Epiblast-restricted Cre expression in MORE mice: a tool to distinguish embryonic vs. extra-embryonic gene function. Genesis 26:113-115.

Townley-Tilson WH, Callis TE, Wang D (2010) MicroRNAs 1, 133, and 206: critical factors of skeletal and cardiac muscle development, function, and disease. Int J Biochem Cell Biol 42:1252-1255.

van den Munckhof P, Luk KC, Ste-Marie L, Montgomery J, Blanchet PJ, Sadikot AF, Drouin J (2003) Pitx3 is required for motor activity and for survival of a subset of midbrain dopaminergic neurons. Development 130:2535-2542.

Velleca MA, Wallace MC, Merlie JP (1994) A novel synapse-associated noncoding RNA. Mol Cell Biol 14:7095-7104.

Zweifel LS, Fadok JP, Argilli E, Garelick MG, Jones GL, Dickerson TM, Allen JM, Mizumori SJ, Bonci A, Palmiter RD (2011) Activation of dopamine neurons is critical for aversive conditioning and prevention of generalized anxiety. Nat Neurosci 14:620-626. 